\title{
Epithelial-mesenchymal transition in chronic rhinosinusitis (CRS) and the prognostic value of $\alpha-S M A$ in postoperative outcomes of patients with CRS
}

\author{
HAN LI $^{1 *}$, QUAN LIU ${ }^{1 *}$, HUAN WANG $^{1 *}$, XI-CAI SUN ${ }^{1}$, HUA-PENG YU $^{1}$, LI HU $^{2}$ and DE-HUI WANG \\ ${ }^{1}$ Department of Otolaryngology; ${ }^{2}$ Experimental Center, Eye Ear Nose and Throat Hospital of Fudan University, \\ Shanghai 200031, P.R. China
}

Received November 23, 2018; Accepted May 28, 2019

DOI: $10.3892 / \mathrm{mmr} .2019 .10461$

\begin{abstract}
Tissue remodeling is the pathological basis of the symptoms encountered in chronic rhinosinusitis (CRS). Epithelial-mesenchymal transition (EMT) may participate in this process. The present study was designed to investigate the involvement of EMT in CRS. In addition, the prognostic value of the EMT biomarker $\alpha$-smooth muscle actin ( $\alpha$-SMA) was assessed in patients with CRS who underwent endoscopic sinus surgery (ESS). A total of 13 patients with CRS without nasal polyps (CRSsNP), 13 patients with CRS with nasal polyps (CRSwNP) and 13 control subjects were enrolled. The expression of EMT markers was determined in sinonasal specimens by qPCR, western blot and immunofluorescence assays. EMT features were evaluated in primary nasal epithelial cells (NECs) with transforming growth factor (TGF)- $\beta 1$ stimulation. The associations were assessed between $\alpha$-SMA expression and the clinical features of CRS. Epithelial and mesenchymal markers were overexpressed in the sinonasal specimens of both CRSsNP and CRSwNP patients. Alterations in the expression pattern were more apparent in the CRSsNP patients. Following incubation of primary NECs with TGF- $\beta 1$, a mesenchymal shape was acquired. In addition, NECs that co-expressed $\alpha$-SMA and cytokeratin were readily detected and the protein levels of $\alpha$-SMA were elevated. In contrast to $\alpha$-SMA, the levels of E-cadherin were decreased. The protein levels of $\alpha$-SMA were negatively correlated with endoscopic scores and several postoperative symptoms. In conclusion, partial EMT occurred in patients with CRS, notably in CRSsNP patients. Moreover, primary NECs could undergo
\end{abstract}

Correspondence to: Professor De-Hui Wang, Department of Otolaryngology, Eye Ear Nose and Throat Hospital of Fudan University, 83 Fenyang Road, Xuhui, Shanghai 200031, P.R. China E-mail: wangdehuient@sina.com

*Contributed equally

Key words: chronic rhinosinusitis, epithelial-mesenchymal transition, nasal epithelial cells, $\alpha$-smooth muscle actin, prognosis
EMT following TGF- $\beta 1$ treatment in vitro. In addition, $\alpha$-SMA could be considered an efficient predictor for postoperative endoscopic and symptomatic outcomes in patients with CRS treated with ESS.

\section{Introduction}

Chronic rhinosinusitis (CRS) is a chronic inflammatory disease involving sinonasal mucosa (1). CRS affects approximately $8 \%$ of the Chinese population, and results in serious health consequences (2). Although endoscopic sinus surgery (ESS) can improve the prognosis of patients with CRS in the case of unsuccessful medical therapy, a refractory course presents in a minority of cases. Therefore, the pathogenesis of CRS requires further investigation.

Epithelial-mesenchymal transition (EMT) is a biological process, in which epithelial cells acquire mesenchymal properties and lose their epithelial phenotype (3). The features of EMT include decreased expression of epithelial makers, such as E-cadherin and cytokeratin, and increased expression of mesenchymal markers, namely $\alpha$-smooth muscle actin $(\alpha-S M A)$, vimentin and fibronectin. EMT plays key roles in crucial cell functions including organogenesis, tumor formation and fibrosis $(4,5)$. Recently, EMT was reported to be involved in a number of airway diseases (6-9). The pathological basis of the symptoms in CRS includes tissue remodeling, which is characteristic of the abnormal deposition of the extracellular matrix (ECM). Currently, a limited number of studies have been conducted that have examined the association between EMT and CRS (10-16). However, these results were controversial and further investigation on this topic is necessary. The evidence from primary nasal epithelial cells (NECs) in patients with CRS remains limited (14-16), whereas the association between EMT markers and clinical features in CRS has been scarcely explored (16). Therefore, the involvement of EMT in CRS is essential for improving the prognosis of these patients.

The present study aimed to examine the expression of EMT markers in sinonasal specimens from CRS and control patients. CRS was divided into two types, namely chronic rhinosinusitis without nasal polyps (CRSsNP) and chronic rhinosinusitis with nasal polyps (CRSwNP) (17). In addition, 
EMT features were evaluated in primary NECs following transforming growth factor (TGF)- $\beta 1$ stimulation. The associations between the expression levels of $\alpha$-SMA and the clinical characteristics of CRS were also determined.

\section{Materials and methods}

Subjects. In the present study, 39 patients were recruited, including 23 females and 16 males, aged 11-72 years old (45.85 14.88$)$. All patients underwent endoscopic sinus surgery (ESS) from September 2009 to February 2011 at the Department of Otolaryngology of the Affiliated Eye Ear Nose and Throat Hospital (AEENTH) of Fudan University. Patients with immunodeficiency diseases (human immunodeficiency virus, diabetes, renal disease) were excluded. CRS subjects were diagnosed according to the EPOS-2007 criteria (17). The patients undergoing ESS for benign diseases other than CRS were employed as the control subjects.

Clinical information was recorded prior to surgery, including age, sex, disease duration, sinus surgical history, smoking history, co-existence of allergic rhinitis, asthma, aspirin sensitivity and gastroesophageal reflux disease (GERD). The Lund-Mackay CT score was estimated preoperatively. The visual analogue scale (VAS), the rhinosinusitis outcome measure-31 (RSOM-31) and the Lund-Kennedy endoscopy scores were assessed pre- and post-operatively as previously described (18-20).

The samples were obtained from the ethmoid sinus with edema during surgery or during discharge. These samples were representative of CRS, whereas the samples from the nasal cavity were used as controls. Each sample was divided into 4 specimens for the detection of mRNA and protein expression, for hematoxylin and eosin (H\&E) staining and for immunofluorescence staining, respectively.

Reverse transcription-quantitative PCR $(q P C R)$. Total RNA was extracted from tissues with TRIzol ${ }^{\circledR}$ reagent (Invitrogen; Thermo Fisher Scientific, Inc.), and subsequently reverse transcribed into cDNA with a SuperScript ${ }^{\mathrm{TM}}$ first strand synthesis kit (Invitrogen; Thermo Fisher Scientific, Inc.). RT-qPCR was performed with a thermal cycler (Applied Biosystems; Thermo Fisher Scientific, Inc.). Amplification conditions for the RT reaction were $42^{\circ} \mathrm{C}$ for $50 \mathrm{~min}$ and $70^{\circ} \mathrm{C}$ for $15 \mathrm{~min}$. qPCR was then performed using an SYBR ExScript kit (Takara Biotechnology Co., Ltd.) and conducted on a Mx3000 Real-Time PCR system (Stratagene, La Jolla, CA). Conditions of the PCR amplification were: $94^{\circ} \mathrm{C}(5 \mathrm{~min})$, then 30 cycles of $94^{\circ} \mathrm{C}$ for $30 \mathrm{sec}, 56^{\circ} \mathrm{C}$ for $30 \mathrm{sec}$ and $72^{\circ} \mathrm{C}$ for $60 \mathrm{sec}$, with a final extension at $72^{\circ} \mathrm{C}$ for $10 \mathrm{~min}$. Each sample was measured in triplicate. The mRNA levels were determined using the $2^{-\Delta \Delta C q}$ method (21). Glyceraldehyde-3-phosphate dehydrogenase $(G A P D H)$ was selected as the control gene. The primer sequences are presented in Table SI.

Western blot analysis. The tissues were incubated with lysis buffer (Beyotime Institute of Biotechnology) on ice. The protein concentration was quantified using a BCA protein concentration assay kit (Beyotime Institute of Biotechnology). The proteins were separated by SDS-PAGE and were loaded on the $10 \%$ gels in equal amounts $(40 \mu \mathrm{g})$. Following electrophoresis, they were transferred to PVDF membranes. The membranes were blocked using 5\% BSA (Beyotime Institute of Biotechnology) for $1 \mathrm{~h}$ at room temperature, and immunoblotted with mouse monoclonal antibodies against $\alpha$-SMA (1:1,000; sc-130617; Santa Cruz Biotechnology, Inc.), TGF- $\beta 1$ (1:1,000; ab64715; Abcam), E-cadherin (1:1,000; ab1416; Abcam), fibronectin (1:1,000; ab26245; Abcam) and GAPDH (1:1,000; cat. no. 97166; Cell Signaling Technology) overnight at $4^{\circ} \mathrm{C}$. The following morning, the membranes were incubated with anti-mouse $\operatorname{IgG}$ conjugated to horseradish peroxidase (1:2,000; cat. no. 7076; Cell Signaling Technology). The blots were visualized by Amersham ECL ${ }^{\mathrm{TM}}$ Select (GE Healthcare Life Sciences) with X-OMAT BT film (Carestream Health). Quantity One 2.0 (Bio-Rad Laboratories, Inc.) was used for the quantification of protein expression. The protein level of control group was set as 1; all data were normalized to the control group.

Immunofluorescence and $H \& E$ staining. The tissue samples were fixed with $4 \%$ paraformaldehyde for $24 \mathrm{~h}$ at room temperature and embedded in paraffin. Embedded tissues were cut into $5-\mu \mathrm{m}$ sections and deparaffinized with xylene and hydrated with a series of ethanol solutions in a decreasing concentration gradient. The sections were permeabilized with $0.1 \%$ Triton $\mathrm{X}-100$, blocked with $1 \%$ BSA for $1 \mathrm{~h}$ at room temperature, and subsequently incubated with primary antibodies overnight at $4^{\circ} \mathrm{C}$, including mouse monoclonal antibodies against TGF- $\beta 1$ (1:500; ab64715; Abcam), matrix metalloproteinase (MMP)-9 (1:500; ab58803; Abcam) and fibronectin $(1: 100 ; a b 26245$; Abcam). The sections were probed with goat anti-mouse cyanine3-labeled secondary antibody (1:400; M30010, Invitrogen; Thermo Fisher Scientific, Inc.) for $2 \mathrm{~h}$ at room temperature, and subsequently mounted with DAPI for $20 \mathrm{~min}$ at room temperature. Fluorescence was measured over 10 fields per sample at magnification, x400 (x40 objective lens) by fluorescence microscopy (Leica Microsystems $\mathrm{GmbH}$ ).

For double-labeling immunofluorescence, the primary antibodies used were the following: mouse monoclonal antibody against $\alpha$-SMA (1:200; sc-130617; Santa Cruz Biotechnology, Inc.) and rabbit monoclonal antibody against pan-cytokeratin (1:200; ab234297; Abcam). FITC-conjugated rabbit anti-mouse (1:100; F9137; Sigma-Aldrich; Merck $\mathrm{KGaA}$ ) and cyanine3-conjugated goat anti-rabbit secondary antibodies (1:200; A10520; Thermo Fisher Scientific, Inc.) were applied respectively.

Furthermore, the pathological classification of Hellquist was conducted for CRSwNP based on H\&E staining, as described previously (22).

Isolation of primary NECs. Each tissue was cut into small pieces and incubated with collagenase for $12-24 \mathrm{~h}$ at $4^{\circ} \mathrm{C}$, as soon as the sinonasal mucosa was removed from the patients with CRS. The suspension was vortexed and filtered $(50 \mu \mathrm{m})$ to remove the clumps. Magnetic cell sorting method was used to obtain NECs. The cells were resuspended in degassed buffer [phosphate-buffered saline (PBS) pH 7.2, 0.5\% FCS, $2 \mathrm{ml}$ EDTA] at a density of $10^{7}$ cells $/ 80 \mu \mathrm{l}$ and incubated with microbeads conjugated to monoclonal mouse anti-human CD326 antibodies (Miltenyi Biotec, Inc.) at a bead-to-total cell 
A

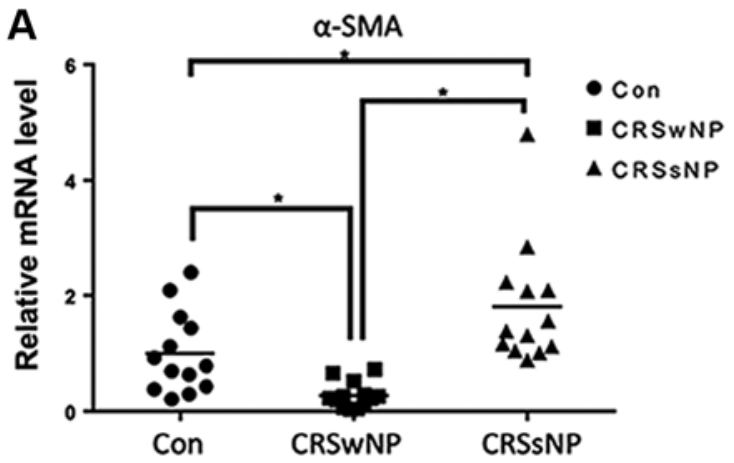

C
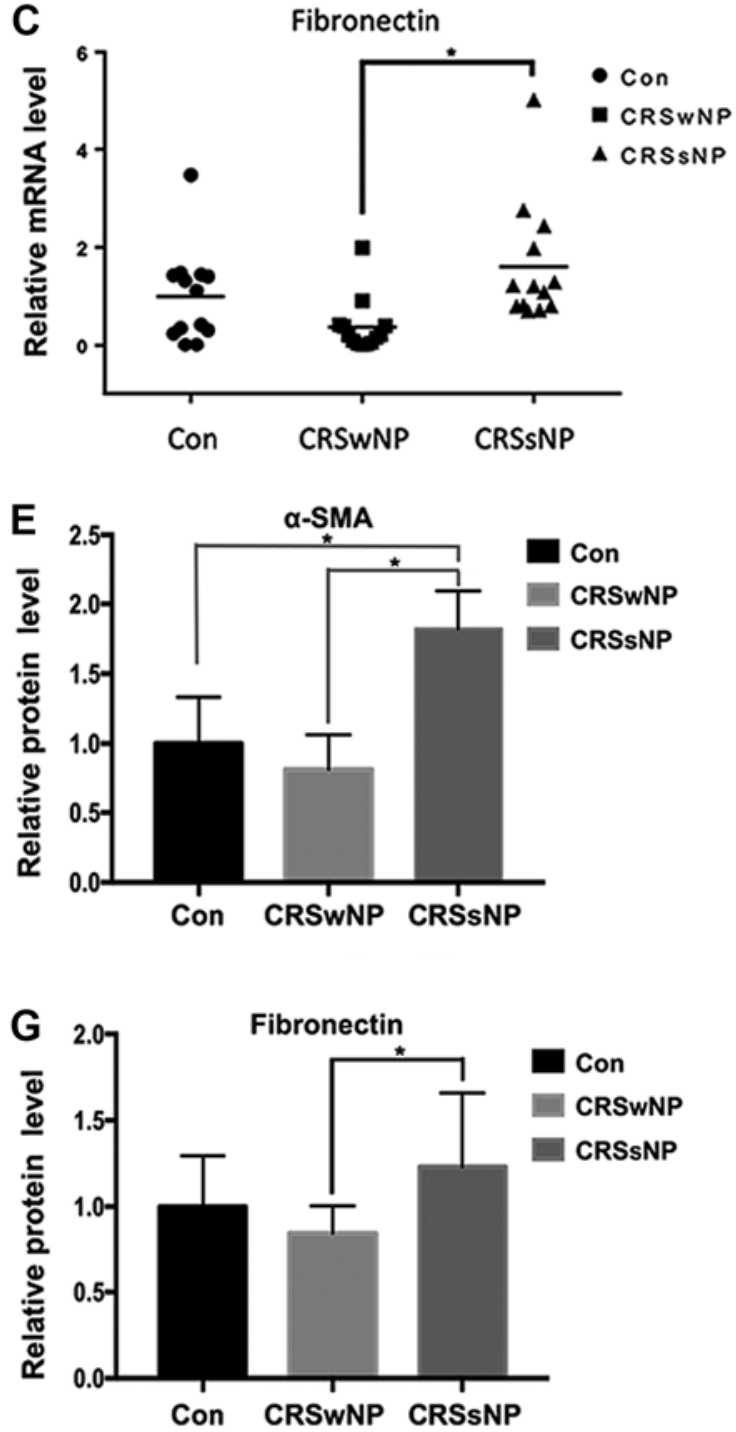

B

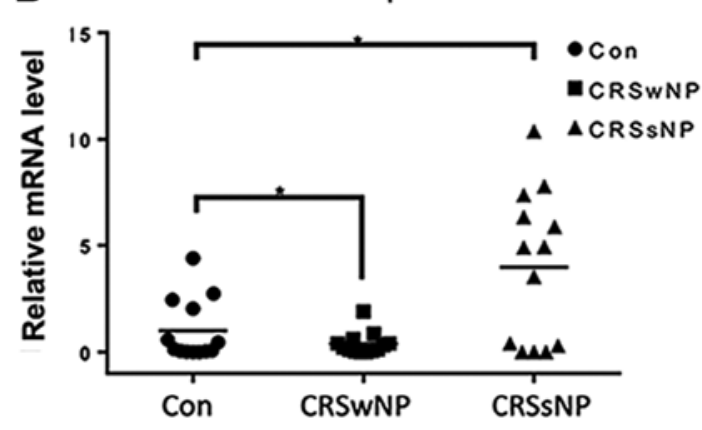

D

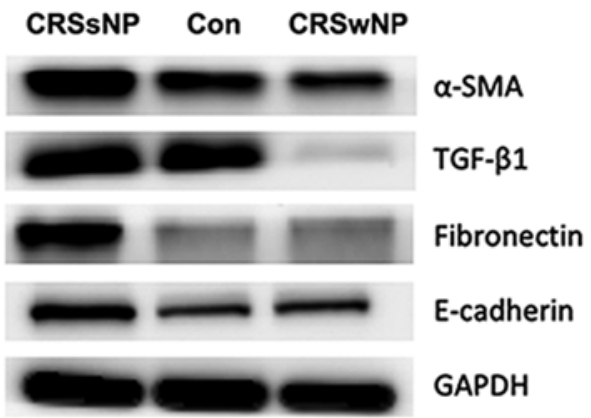

$\mathbf{F}$
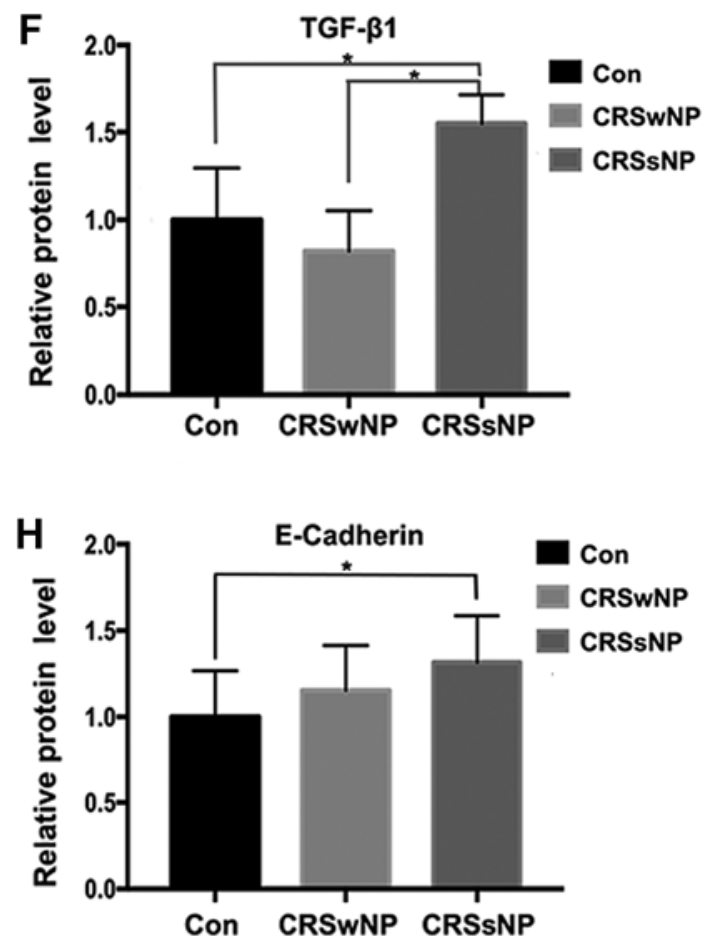

Figure 1. Relative mRNA and protein levels of EMT markers in CRSsNP, CRSwNP and control subjects. (A-C) Relative mRNA levels of $\alpha-S M A, T G F-\beta 1$, and fibronectin in the three groups. (D) Protein levels of EMT markers in the three groups. (E-H) Quantified data of western blot analysis. "P<0.05. EMT, epithelial-mesenchymal transition; CRS, chronic rhinosinusitis; $\alpha$-SMA, $\alpha$-smooth muscle actin; TGF- $\beta 1$, transforming growth factor- $\beta 1$; CON, control group; CRSsNP, CRS without nasal polyps; CRSwNP, CRS with nasal polyps.

ratio of $20 \mu \mathrm{l}: 10^{7}$ cells for $20 \mathrm{~min}$ at $4^{\circ} \mathrm{C}$. The supernatant was aspirated by collecting the beads using an MS Column and a MACS Separator (Miltenyi Biotec, Inc.). The cells were resuspended in BEBM media and seeded in a culture flask (Fig. S1A).

The purity of NECs was verified via two methods. First, immunofluorescence was performed on cells, which were fixed and stained with pan-cytokeratin as described above.
The proportions of the NECs (cytokeratin-positive cells) in ten fields were recorded under a fluorescence microscope. Second, flow cytometry was performed. Cells were digested with $0.25 \%$ trypsin at $37^{\circ} \mathrm{C}$ for $5 \mathrm{~min}$ and collected via centrifugation at $1,000 \mathrm{x}$ g for $5 \mathrm{~min}$ at $4^{\circ} \mathrm{C}$. Following two washes with ice-cold PBS, the cells were re-suspended in $50 \mu 1$ of PBS, blocked with anti-human IgG (1:1,000; cat. no. ab195574; Abcam) at 
A

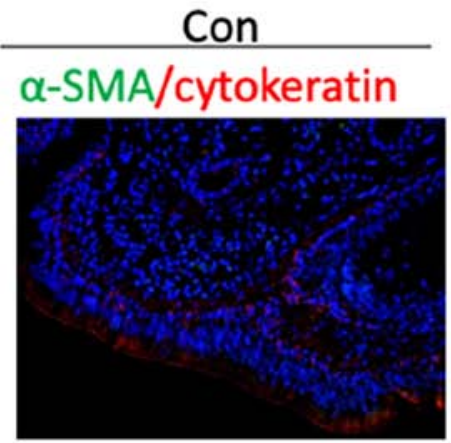

B TGF- $\beta 1$

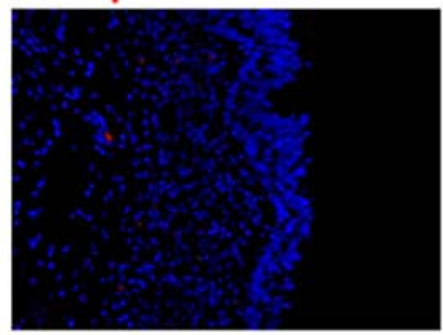

C MMP-9

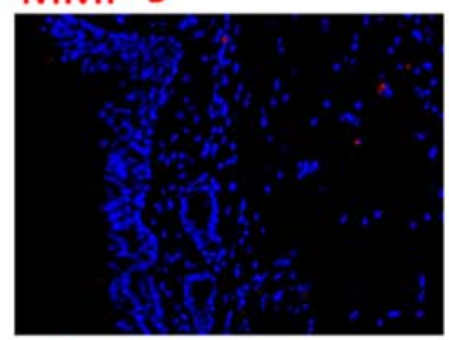

D Fibronectin

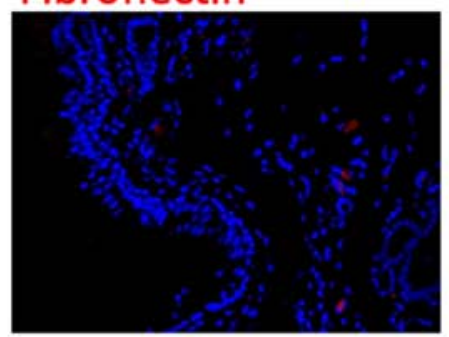

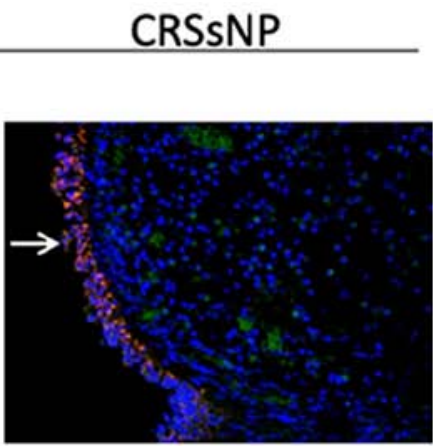
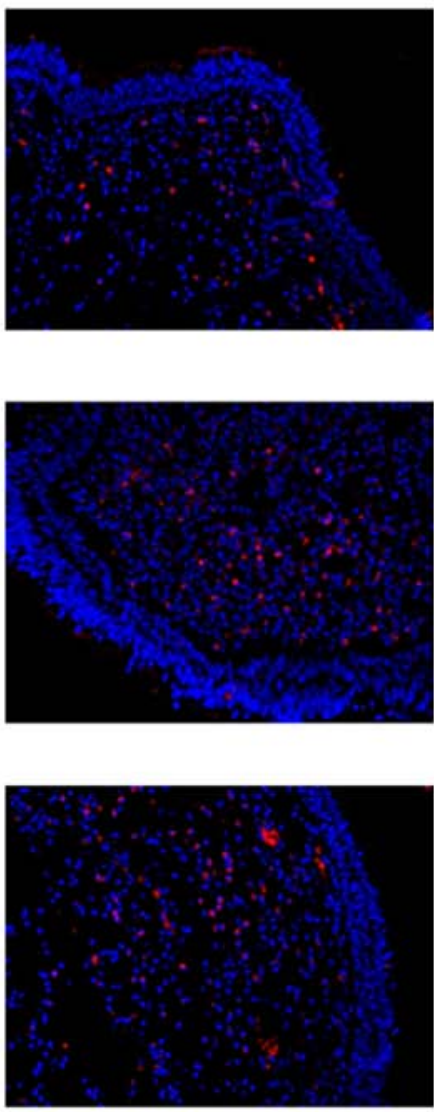
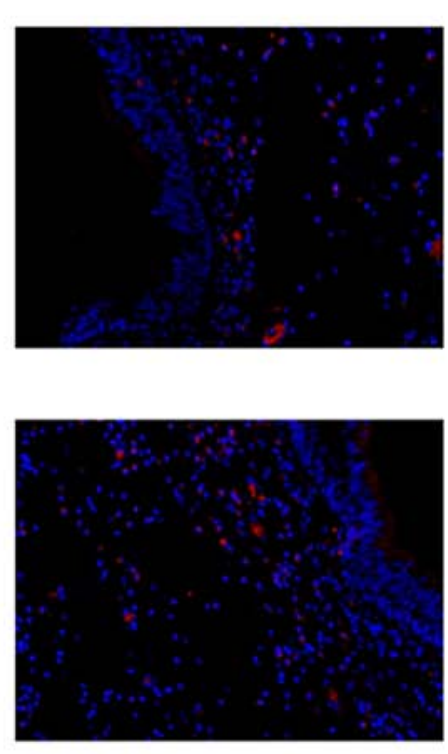

CRSWNP
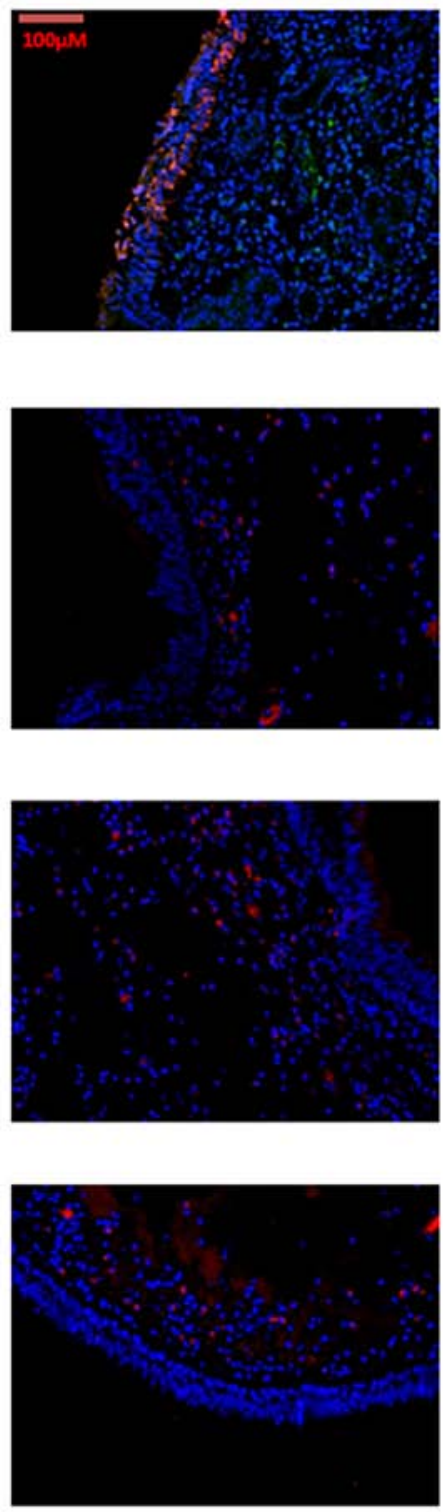

Figure 2. Immunofluorescence staining of the tissues from CRSsNP, CRSwNP and the control subjects. (A) Red fluorescence labeling denotes $\alpha$-SMA, green fluorescence labeling keratin and yellow fluorescence is indicative of the cells in the EMT process (arrow). (B) Red fluorescence labeling of TGF- $\beta 1$. (C) Red fluorescence labeling of MMP-9. (D) Red fluorescence labeling of fibronectin. EMT, epithelial-mesenchymal transition; CRS, chronic rhinosinusitis; $\alpha$-SMA, $\alpha$-smooth muscle actin; TGF- $\beta 1$, transforming growth factor- $\beta 1$; MMP-9, matrix metallopeptidase 9 ; CON, control group; CRSsNP, CRS without nasal polyps; CRSwNP, CRS with nasal polyps.

room temperature for $15 \mathrm{~min}$, and subsequently incubated with mouse phycoerythrin-conjugated anti-pan-cytokeratin (1:1,000; cat. no. SAB4700668; Sigma-Aldrich; Merck $\mathrm{KGaA}$ ) for $30-45 \mathrm{~min}$ at $4^{\circ} \mathrm{C}$ in the dark. The NECs were identified using an EPICS XL flow cytometer (Beckman Coulter, Inc.) and analyzed by Cell Quest software version FCS2.0 (BD Biosciences). Cytokeratin-positive cells were considered NECs. The purity of the NECs reached $89.3 \%$ by immunofluorescence or $92.1 \%$ by flow cytometry, respectively (Fig. S1B and C).

EMT induction and assessment. For EMT induction, the cells were incubated with TGF- $\beta 1(1,5$ and $10 \mathrm{ng} / \mathrm{ml})$ for 1,2 and 3 days, respectively. The morphology of the cells was observed using a phase contrast microscope. Double-labeling immunofluorescence of $\alpha$-SMA and cytokeratin was applied. The expression levels of E-cadherin and $\alpha$-SMA were determined by western blot analysis, as described above.

Statistical analysis. Statistical analyses were carried out using SPSS 14.0 (SPSS, Inc.). The data are presented as mean \pm standard deviations $(\mathrm{SD})$. The differences were analyzed by one-way ANOVA followed by Bonferroni post hoc test among multiple groups. The Kruskal-Wallis test was employed for data with skewed distribution. The correlations between $\alpha$-SMA levels and clinical parameters of CRS were calculated by the Pearson correlation method. $\mathrm{P}<0.05$ was used to indicate significant differences. 
A

1 day

3 days

5 days

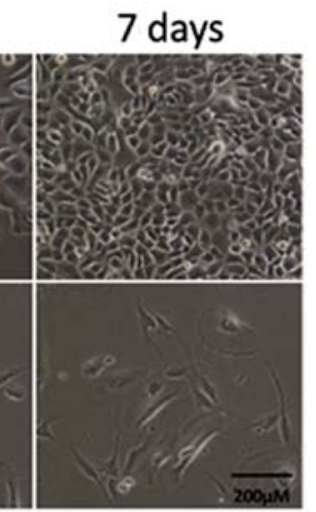

B
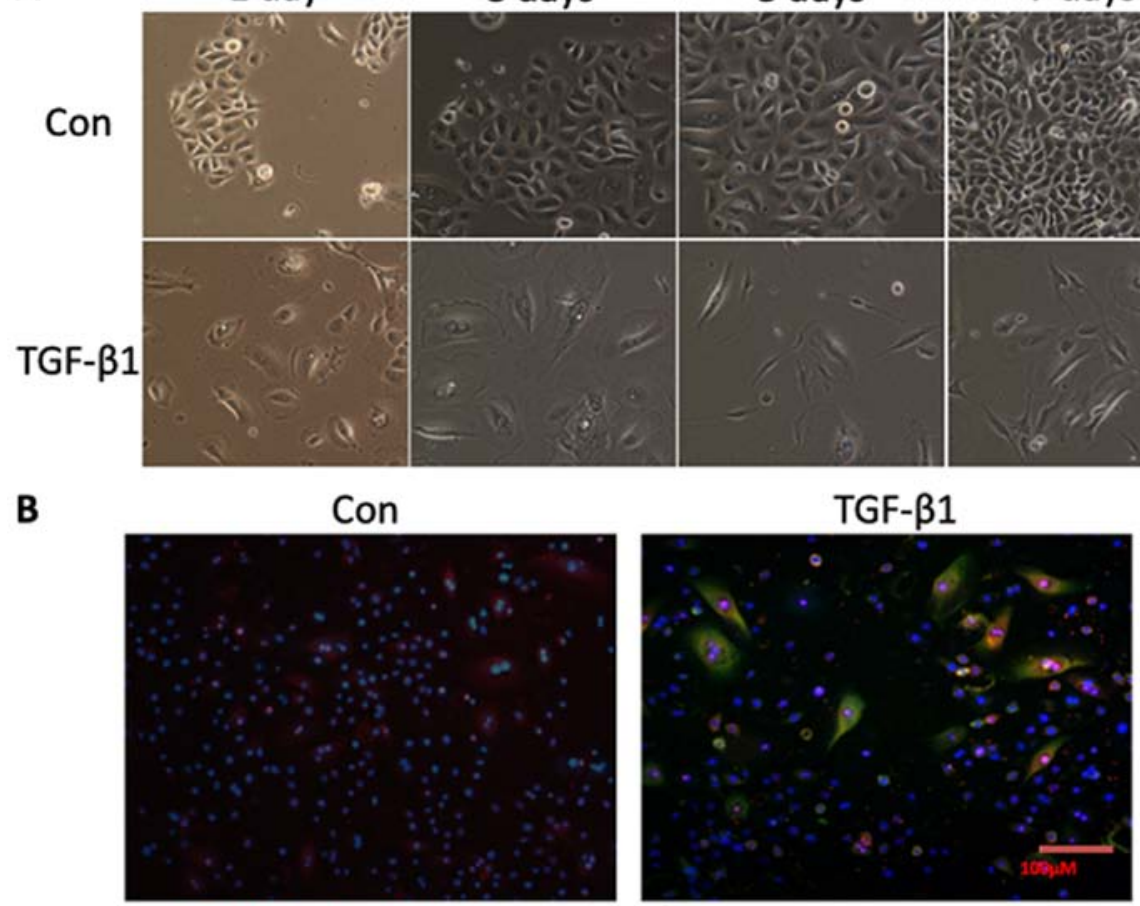

$\alpha-S M A / c y t o k e r a t i n$
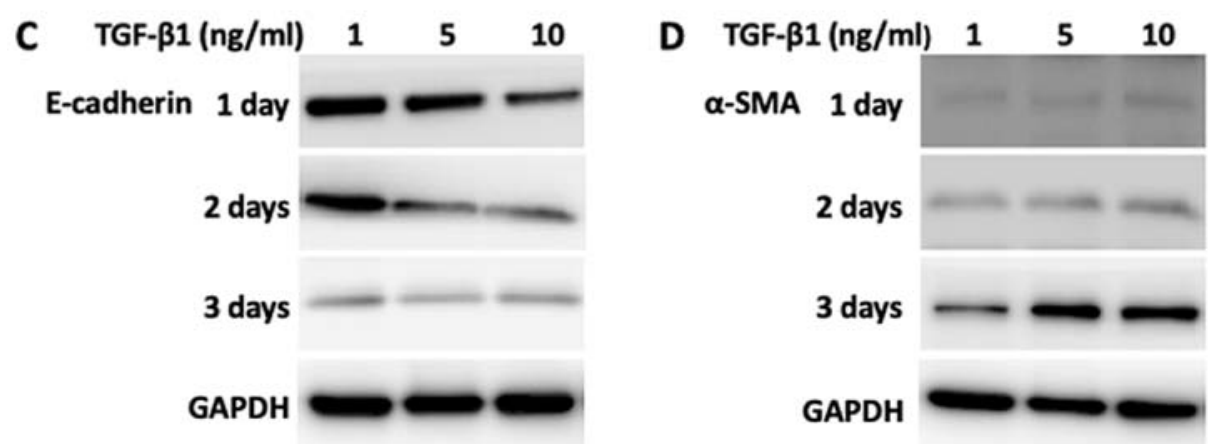

Figure 3. Induction of EMT in NECs that were treated with TGF- $\beta 1$. (A) Phase-contrast images of NECs with or without TGF- $\beta 1$ treatment on day $1,3,5$ and 7. (B) Immunofluorescence staining of $\alpha$-SMA (red) and cytokeratin (green). (C) Western blot analysis for E-cadherin on day 1, 2 and 3. (D) Western blot analysis for $\alpha$-SMA levels on day 1,2 and 3. EMT, epithelial-mesenchymal transition; NEC, nasal epithelial cells; $\alpha$-SMA, $\alpha$-smooth muscle actin; TGF- $\beta 1$, transforming growth factor- $\beta 1$; $\mathrm{CON}$, control group.

\section{Results}

Basic characteristics. A total of 26 patients with CRS and 13 control subjects were enrolled in the present study. Patients with CRS included $13 \mathrm{CRSwNP}$ and $13 \mathrm{CRSsNP}$ cases. The controls involved 3 cases with paranasal sinus cyst, 3 cases with cerebrospinal fluid rhinorrhea, 2 cases with nasal septum deviation, 2 cases with traumatic optic neuropathy, 1 case with pituitary adenoma, 1 case with thyroid eye disease and 1 case with frontal sinus osteoma. The basic characteristics are documented in Table SII.

Expression of EMT markers in tissues of patients with CRS and control subjects. The mRNA levels of E-cadherin in CRS group were significantly increased compared with in the control group, whereas, no significant differences were observed between the two groups in the mRNA levels of other markers including $\alpha$-SMA, TGF- $\beta 1$, MMP-9, fibronectin and vimentin (Table SIII). The mRNA levels of $E$-cadherin, $\alpha$-SMA and $T G F-\beta 1$ in CRSsNP were significantly higher than those noted in the control subjects. CRSwNP exhibited decreased mRNA levels of $\alpha$-SMA and vimentin compared with the expression levels of these markers in the control subjects. CRSsNP tissues had elevated expression of E-cadherin, $\alpha$-SMA, TGF- $\beta 1$, fibronectin and vimentin when compared with CRSwNP. MMP-9 mRNA levels exhibited no difference between these two groups (Fig. 1A-C).

The protein levels of E-cadherin in CRS were significantly higher than those noted in the control subjects. The protein levels of $\alpha$-SMA, TGF- $\beta 1$ and E-cadherin in CRSsNP were significantly increased compared with the control group. The protein levels of $\alpha$-SMA, TGF- $\beta 1$ and fibronectin in CRSsNP were significantly upregulated compared with CRSwNP (Fig. 1D-H). The protein levels of the EMT markers are presented in Table SIV. 
Table I. Correlation analysis between $\alpha$-SMA and CRS characteristics.

\begin{tabular}{|c|c|c|c|}
\hline \multirow[b]{2}{*}{ Item } & \multirow[b]{2}{*}{ Proportion or mean \pm SD } & \multicolumn{2}{|c|}{ Correlation with $\alpha$-SMA (WB) } \\
\hline & & $\mathrm{r}$ & P-value \\
\hline Age (years) & $46.04 \pm 15.82$ & -0.280 & 0.167 \\
\hline $\operatorname{Sex}(M / F)$ & $16 / 10$ & -0.270 & 0.183 \\
\hline Course of CRS (years) & $7.06 \pm 6.63$ & -0.223 & 0.273 \\
\hline Allergic rhinitis (+/-) & $11 / 15$ & 0.013 & 0.952 \\
\hline Asthma (+/-) & $2 / 24$ & -0.281 & 0.165 \\
\hline Smoking (+/-) & $7 / 19$ & -0.402 & 0.042 \\
\hline Aspirin allergy (+/-) & $0 / 26$ & / & / \\
\hline Gastroesophageal reflux (+/-) & $2 / 24$ & 0.018 & 0.931 \\
\hline History of sinus surgery (+/-) & $3 / 23$ & -0.363 & 0.068 \\
\hline
\end{tabular}

CRS, chronic rhinosinusitis; $\alpha$-SMA, $\alpha$-smooth muscle actin; WB, western blotting; M, male; F, female; +, with; -, without.

The protein levels of $\alpha$-SMA, TGF- $\beta 1$, fibronectin and MMP-9 were increased in CRSsNP, as demonstrated by immunofluorescence assays (Fig. 2). In addition, the cells that co-expressed $\alpha$-SMA and cytokeratin were detected in the mucosal epithelium in both CRSsNP and CRSwNP using double-labeling immunofluorescence assays (Fig. 2A).

Characteristics of EMT in primary NECs following TGF- $\beta 1$ treatment. Following incubation with TGF- $\beta 1$, the NECs acquired a mesenchymal phenotype as demonstrated by phase contrast microscopy. After $24 \mathrm{~h}$ of induction by TGF- $\beta 1$, the junctions among epithelial cells were interrupted, and the cells became dispersed and flat. After 3 days, the cytoplasm began to show polarity, extending in two opposite directions like tentacles. After 7 days, over half of the cells presented typical long spindle fibroblasts (Fig. 3A). In addition, NECs that co-expressed $\alpha$-SMA and cytokeratin were detected following TGF- $\beta 1$ treatment by double-labeling immunofluorescence staining (Fig. 3A and B). In addition, the protein levels of $\alpha$-SMA were increased, whereas the levels of E-cadherin were decreased following TGF- $\beta 1$ treatment (Fig. 3C).

Correlation between $\alpha$-SMA protein expression and clinical features of $C R S$. A negative correlation was evident between $\alpha$-SMA protein expression and smoking. No association existed between $\alpha$-SMA levels and the remaining clinical characteristics (Table I).

With regard to preoperative parameters, no correlation was noted between $\alpha$-SMA levels and the symptoms noted in RSOM-31 (Table SV). The $\alpha$-SMA levels were inversely correlated with the Lund-Kennedy score, while they did not correlate with specific endoscopic signs (Table II). $\alpha$-SMA levels were negatively correlated with CT scores of anterior ethmoid sinus, while no correlation was noted with the other paranasal sinuses, indicating that CRS simply involving anterior ethmoid were less likely to undergo EMT (Table SVI).

Correlation between $\alpha$-SMA protein expression and surgical outcomes of CRS. The levels of $\alpha$-SMA were negatively correlated with RSOM-31 symptoms including hyposmia, hearing
Table II. Correlation analysis between $\alpha$-SMA and preoperative endoscopic scores of CRS.

\begin{tabular}{lccc}
\hline & & \multicolumn{2}{c}{$\begin{array}{c}\text { Correlation with } \\
\alpha-S M A\end{array}$} \\
\cline { 3 - 4 } Item & Mean score \pm SD & $r$ & P-value \\
\hline Polyps & $3.19 \pm 1.47$ & -0.082 & 0.691 \\
Edema & $3.42 \pm 0.99$ & -0.335 & 0.095 \\
Discharge & $3.23 \pm 1.11$ & -0.363 & 0.068 \\
Scarring/adhesion & $0.04 \pm 0.20$ & 0.133 & 0.518 \\
Crusting & $0.23 \pm 0.65$ & -0.369 & 0.064 \\
Endoscopic score & $10.12 \pm 2.61$ & -0.408 & 0.038 \\
\hline
\end{tabular}

CRS, chronic rhinosinusitis; $\alpha$-SMA, $\alpha$-smooth muscle actin.

loss, fatigue, and reduced productivity (Table III). A trend towards negative correlation was observed between $\alpha$-SMA levels and VAS score, although no significance was found. Negative correlations were present between $\alpha$-SMA levels and endoscopic score, and between $\alpha$-SMA levels and endoscopic signs, including polyps, discharge and crusting (Table IV).

\section{Discussion}

Chronic rhinosinusitis (CRS) is a prevalent disease in otolaryngology. It affects the quality of life (QOL) of patients and imposes a heavy burden on socioeconomic resources (23). The abnormal deposition of extracellular matrix (ECM) in the mucosa is one of the pathological causes of CRS. Epithelial-mesenchymal transition (EMT) plays crucial roles in tissue remodeling in several pathophysiological processes (24). Recently, the induction of EMT was reported in CRS by several studies (10-16). Nevertheless, inconsistent results were provided. A limited number of studies have indicated that EMT is involved in patients with CRS with nasal polyps (CRSwNP) and not in patients with CRS without 
Table III. Correlation analysis between $\alpha$-SMA and postoperative symptom scores of the patients with CRS.

\begin{tabular}{|c|c|c|c|}
\hline \multirow[b]{2}{*}{ Item } & \multirow[b]{2}{*}{ Mean score \pm SD } & \multicolumn{2}{|c|}{ Correlation with $\alpha$-SMA } \\
\hline & & $\mathrm{r}$ & P-value \\
\hline VAS score & $22.77 \pm 20.41$ & -0.364 & 0.067 \\
\hline RSOM - 31 score & $43.04 \pm 42.86$ & -0.343 & 0.086 \\
\hline Rhinobyon & $2.27 \pm 2.75$ & -0.233 & 0.252 \\
\hline Non-purulent nasal mucus & $2.46 \pm 2.39$ & -0.190 & 0.354 \\
\hline Sneezing & $1.42 \pm 1.98$ & 0.024 & 0.909 \\
\hline Olfactory decline & $4.27 \pm 5.77$ & -0.472 & 0.015 \\
\hline Nose backflow & $1.19 \pm 1.98$ & 0.016 & 0.937 \\
\hline Purulent nasal mucus & $1.69 \pm 2.92$ & 0.349 & 0.081 \\
\hline Eye itching & $1.19 \pm 2.71$ & 0.246 & 0.225 \\
\hline Sore eyes & $0.54 \pm 1.14$ & 0.103 & 0.618 \\
\hline Difficulty falling asleep & $0.69 \pm 1.93$ & -0.161 & 0.432 \\
\hline Night awakening & $0.65 \pm 2.37$ & -0.249 & 0.220 \\
\hline Poor sleep & $1.42 \pm 2.34$ & -0.300 & 0.136 \\
\hline Wake up tired & $1.35 \pm 1.98$ & -0.153 & 0.456 \\
\hline Ear swelling & $0.50 \pm 1.66$ & -0.240 & 0.237 \\
\hline Tinnitus & $2.58 \pm 5.12$ & -0.372 & 0.061 \\
\hline Dizziness & $0.69 \pm 1.74$ & -0.261 & 0.198 \\
\hline Ear pain & $0.46 \pm 1.66$ & -0.232 & 0.255 \\
\hline Hearing loss & $1.73 \pm 3.65$ & -0.413 & 0.036 \\
\hline Weak & $2.15 \pm 2.63$ & -0.537 & 0.005 \\
\hline Loss of efficiency & $0.88 \pm 1.90$ & -0.420 & 0.033 \\
\hline Lose focus & $1.38 \pm 2.86$ & -0.030 & 0.884 \\
\hline Headache & $0.88 \pm 2.76$ & -0.037 & 0.859 \\
\hline Facial pain & $0.23 \pm 0.51$ & -0.301 & 0.135 \\
\hline Cough & $1.31 \pm 1.83$ & 0.230 & 0.259 \\
\hline Dyspnea & $0.46 \pm 0.91$ & -0.085 & 0.678 \\
\hline Need tissue frequently & $2.69 \pm 3.11$ & -0.121 & 0.556 \\
\hline Frequently rubbing nose and eyes & $1.42 \pm 3.24$ & -0.039 & 0.849 \\
\hline Often blow nose & $3.00 \pm 3.11$ & -0.229 & 0.260 \\
\hline Ozostomia & $0.96 \pm 1.59$ & 0.072 & 0.728 \\
\hline Irritable & $1.15 \pm 1.91$ & -0.270 & 0.183 \\
\hline Frustrated & $0.62 \pm 1.55$ & -0.017 & 0.934 \\
\hline Embarrassed & $0.77 \pm 1.61$ & -0.206 & 0.313 \\
\hline
\end{tabular}

CRS, chronic rhinosinusitis; $\alpha$-SMA, $\alpha$-smooth muscle actin.

polyps (CRSsNP) (10-12), while Hupin et al demonstrated increased vimentin expression levels in both groups (16). The majority of these studies explored the association of CRSwNP with the aforementioned markers, while a limited number of studies focused on CRSsNP. Therefore, additional evidence is required to unravel the involvement of EMT markers in CRSsNP. In the present study, the cells were stained using double-labeling with $\alpha$-SMA and cytokeratin, indicating the induction of EMT in CRS. To the best of our knowledge, this is the first study that utilized double-label staining to assess EMT induction in CRS. Double-label staining is an intuitive evidence for EMT that illustrates whether cells are undergoing this process. Several double-label stained cells were observed in both CRSsNP and CRSwNP, although the fluorescence noted was weak in CRSwNP. EMT can elicit loss of epithelial markers and gain of mesenchymal markers. Increased expression of epithelial (E-cadherin) and mesenchymal markers ( $\alpha$-SMA, vimentin, fibronectin, MMP- 9 ) was noted in both CRSsNP and CRSwNP. This is inconsistent with the findings of Konnecke et al suggesting that the induction of EMT was partially present in CRS (15). Partial EMT was proposed in oncology to describe an intermediate stage of EMT, which generates cancer cells with a higher degree of malignancy than those with a complete EMT phenotype $(25,26)$. However, the underlying mechanisms require further investigation.

In contrast to previous studies, the present report indicated different EMT features in CRSsNP than those noted in CRSwNP, which may be attributed to histological distinctions. 
Table IV. Correlation analysis between $\alpha$-SMA and postoperative endoscopic scores of the patients with CRS.

\begin{tabular}{lccc}
\hline & & \multicolumn{2}{c}{$\begin{array}{c}\text { Correlation with } \\
\alpha \text {-SMA }\end{array}$} \\
\cline { 3 - 4 } Item & Mean score \pm SD & $\mathrm{r}$ & P-value \\
\hline Polyps & $0.19 \pm 0.57$ & -0.439 & 0.025 \\
Edema & $0.65 \pm 0.94$ & 0.129 & 0.529 \\
Discharge & $1.19 \pm 1.30$ & -0.421 & 0.032 \\
Scarring/adhesion & $0.00 \pm 0.00$ & $/$ & $/$ \\
Crusting & $0.81 \pm 0.98$ & -0.709 & $<0.001$ \\
Endoscopic score & $2.81 \pm 2.23$ & -0.640 & $<0.001$
\end{tabular}

CRS, chronic rhinosinusitis; $\alpha$-SMA, $\alpha$-smooth muscle actin.

Edematous and fibrous types are common histological patterns of nasal polyps (NPs) (22). Edematous NPs exhibit a thin layer of epithelium and a lower number of fibroblasts in ECM compared with those noted in fibrous NPs. In the present study, edematous and fibrous types comprised 84.6 and $15.4 \%$ of the NPs, respectively (Fig. S2). Since pathological classification was not conducted in the previous studies, the corresponding comparisons with the present study could not be performed. This suggests that further investigations are required for a more accurate conclusion (10-12).

Although EMT was observed in the nasal epithelial cells (NECs), several studies conducted previously used cell lines as models instead of primary NECs (11-13). Moreover, certain studies have investigated NECs derived from NPs (10), and reports on primary NECs from CRSsNP remain limited (14-16). The incidence of CRSsNP is higher than that of CRSwNP and the two types of CRS may have distinct pathogenic mechanisms. Therefore, it is of great significance to understand the pathogenesis of CRSsNP. We investigated EMT-related features including morphology and expression of the relevant markers in primary NECs. Following TGF- $\beta 1$ exposure, NECs acquired a mesenchymal shape. In addition, NECs co-expressing $\alpha$-SMA and cytokeratin were detected and TGF- $\beta 1$ was able to induce $\alpha$-SMA expression and inhibit E-cadherin expression in NECs. The present study validated that NECs could respond to TGF- $\beta 1$ in a time dependent manner, which is consistent with previous research (15). Taken collectively, the data demonstrated that primary NECs from CRS were able to undergo EMT in response to TGF- $\beta 1$ treatment.

The TGF- $\beta 1$ pathway plays a key role in EMT of many pathological conditions (27). TGF- $\beta 1$ regulates EMT through canonical or non-canonical pathways. The canonical pathway is the SMAD signaling pathway. When TGF- $\beta 1$ binds to TGF- $\beta$ receptor II (TGF $\beta$ R II), the cascade signaling is initiated. TGF $\beta R$ II recruits and phosphorylates TGF $\beta R$ I. TGF $\beta$ R I then phosphorylates R-Smad which is a complex formed with Smad2 and Smad3. Phosphorylated R-Smad forms Co-Smad by binding to Smad4, which then shuttles into the nucleus to regulate transcription via binding to target genes (28). The non-canonical pathways are triggered when the TRRI/II complex activates certain signals such as MAPK, p38 or JNK pathways (27). Currently, it has not been determined whether canonical or non-canonical TGF- $\beta 1$ pathways are implicated in EMT of CRS, which warrants further investigation in the future.

The association between EMT markers and clinical parameters in CRS has been scarcely investigated. Hupin et al reported that increased expression of vimentin was associated with CT score (16). Therefore, the investigation of the association of EMT markers with the clinical features of CRS is helpful for the treatment of such patients. In the present study, partial EMT was noted in the sinus mucosa of patients with CRS, which exhibited a different expression pattern of epithelial markers compared with that noted in the typical EMT process. In sinonasal mucosa, fibroblast markers, such as fibronectin and vimentin are expressed not only in fibroblasts, but also in endothelial cells, while the $\alpha$-SMA protein is specifically expressed in myofibroblasts (29). Therefore, we assessed the correlation between $\alpha$-SMA levels and clinical characteristics of patients with CRS. In the present study, no association was noted between $\alpha$-SMA levels and total CT score. It is interesting to note that a negative correlation was found between $\alpha$-SMA levels and CT score of the anterior ethmoids, suggesting that CRS involving anterior ethmoid was less likely to undergo EMT. This may be related to the anatomical differences of the tissues, since natural drainage of the anterior ethmoid is usually better than that of the other paranasal sinuses. Furthermore, this is the first study to evaluate the prognostic value of the EMT marker $\alpha$-SMA in the surgical outcomes of CRS subjects. Although $\alpha$-SMA was not associated with preoperative symptoms, it was negatively correlated with postoperative symptoms including hyposmia, hearing loss, fatigue and reduced productivity. These were systemic symptoms rather than nasal symptoms, suggesting that the use of $\alpha$-SMA as a marker was more relevant to the overall QOL of CRS following surgery. With regard to the endoscopic parameters, $\alpha$-SMA levels were negatively correlated with preoperative and postoperative scores, and with postoperative signs including polyps, discharge and crusting. These unexpected results provided the first preliminary evidence showing that EMT could predict the surgical outcome of CRS subjects. This hypothesis requires verification using larger sample sizes. According to our clinical experience, the prognosis of CRSsNP is better than that of CRSwNP as a whole, which might be an explanation for the degree of EMT noted in CRSsNP compared with that of CRSwNP.

In conclusion, it was demonstrated that partial EMT occurred in CRS in vivo and that primary NECs responded to TGF- $\beta 1$ induction in vitro, indicating that EMT is involved in the pathogenesis of CRS. In addition, we demonstrated that $\alpha$-SMA could be a predictor for improved postoperative endoscopic and symptomatic outcomes in CRS. These findings require further validation using a large cohort study.

\section{Acknowledgements}

Not applicable.

\section{Funding}

The present study was supported by the National Natural Science Foundation of China (grant nos. 8187040939 and 81600783). 


\section{Availability of data and materials}

All the data generated and analyzed in the present study are available from the corresponding author upon reasonable request.

\section{Authors' contributions}

HL, XCS and DHW conceived and designed the experiments. HL, HW and QL performed the experiments. LH, HPY and QL analyzed the data and conducted the statistical analysis. HL and HW wrote the manuscript. All the authors read and approved the final version of the manuscript and agree to be accounTable for all aspects of the research in ensuring that the accuracy or integrity of any part of the work are appropriately investigated and resolved.

\section{Ethics approval and consent to participate}

The present study was approved by the Ethics Committee of AEENTH and written informed consent was obtained from every patient.

\section{Patient consent for publication}

Not applicable.

\section{Competing interests}

The authors declare that they have no competing interests.

\section{References}

1. Bhattacharyya $\mathrm{N}$ and Gilani S: Prevalence of potential adult chronic rhinosinusitis symptoms in the United States. Otolaryngol Head Neck Surg 159: 522-525, 2018.

2. Shi JB, Fu QL, Zhang H, Cheng L, Wang YJ, Zhu DD, Lv W, Liu SX, Li PZ, Ou CQ and Xu G: Epidemiology of chronic rhinosinusitis: Results from a cross-sectional survey in seven Chinese cities. Allergy 70: 533-539, 2015.

3. Lee JM, Dedhar S, Kalluri R and Thompson EW: The epithelial-mesenchymal transition: New insights in signaling, development, and disease. J Cell Biol 172: 973-981, 2006.

4. Nieto MA, Huang RY, Jackson RA and Thiery JP: EMT: 2016. Cell 166: 21-45, 2016.

5. Stone RC, Pastar I, Ojeh N, Chen V, Liu S, Garzon KI and Tomic-Canic M: Epithelial-mesenchymal transition in tissue repair and fibrosis. Cell Tissue Res 365: 495-506, 2016.

6. Jiang B, Guan Y, Shen HJ, Zhang LH, Jiang JX, Dong XW, Shen $\mathrm{HH}$ and $\mathrm{Xie} \mathrm{QM}$ : Akt/PKB signaling regulates cigarette smoke-induced pulmonary epithelial-mesenchymal transition. Lung Cancer 122: 44-53, 2018.

7. Stasikowska-Kanicka O, Wagrowska-Danilewicz M and Danilewicz M: Immunohistochemical study EMT-related proteins in HPV-, and EBV-negative patients with sinonasal tumours. Pathol Oncol Res 22: 781-788, 2016.

8. Lai T, Li Y, Chen M, Pan G, Wen X, Mai Z, Yuan Y, Lv Y, Lv Q, Cen R, et al: Heparin-binding epidermal growth factor contributes to COPD disease severity by modulating airway fibrosis and pulmonary epithelial-mesenchymal transition. Lab Invest 98:1159-1169, 2018.
9. Sun J, Gu X, Wu N, Zhang P, Liu Y and Jiang S: Human antigen R enhances the epithelial-mesenchymal transition via regulation of ZEB-1 in the human airway epithelium. Respir Res 19: 109, 2018.

10. Shin HW, Cho K, Kim DW, Han DH, Khalmuratova R, Kim SW, Jeon SY, Min YG, Lee CH, Rhee CS and Park JW: Hypoxia-inducible factor 1 mediates nasal polypogenesis by inducing epithelial-to-mesenchymal transition. Am J Respir Crit Care Med 185: 944-954, 2012.

11. Lee M, Kim DW, Yoon H, So D, Khalmuratova R, Rhee CS, Park JW and Shin HW: Sirtuin 1 attenuates nasal polypogenesis by suppressing epithelial-to-mesenchymal transition. J Allergy Clin Immunol 137: 87-98.e7, 2016.

12. Razali RA, Nik Ahmad Eid NAH, Jayaraman T, Amir Hassan MA, Azlan NQ, Ismail NF, Sainik N, Yazid MD, Lokanathan Y, Saim AB and Hj Idrus RB: The potential of Olea europaea extracts to prevent TGF $\beta 1$-induced epithelial to mesenchymal transition in human nasal respiratory epithelial cells. BMC Complement Altern Med 18: 197, 2018.

13. Yang HW,LeeSA, Shin JM,Park IH and Lee HM: Glucocorticoids ameliorate TGF- $\beta 1$-mediated epithelial-to-mesenchymal transition of airway epithelium through MAPK and Snail/Slug signaling pathways. Sci Rep 7: 3486, 2017.

14. Park IH, Kang JH, Shin JM and Lee HM: Trichostatin A inhibits epithelial mesenchymal transition induced by TGF- $\beta 1$ in Airway Epithelium. PLoS One 11: e0162058, 2016.

15. Konnecke M, Burmeister M, Pries R, Boscke R, Bruchhage KL, Ungefroren H, Klimek L and Wollenberg B: Epithelialmesenchymal transition in chronic rhinosinusitis: Differences revealed between epithelial cells from nasal polyps and inferior turbinates. Arch Immunol Ther Exp (Warsz) 65: 157-173, 2017

16. Hupin C, Gohy S, Bouzin C, Lecocq M, Polette M and Pilette C: Features of mesenchymal transition in the airway epithelium from chronic rhinosinusitis. Allergy 69: 1540-1549, 2014.

17. Fokkens W, Lund V, Mullol J and European Position Paper on Rhinosinusitis and Nasal Polyps group: European position paper on rhinosinusitis and nasal polyps 2007. Rhinol Suppl 20: 1-136, 2007.

18. Zhang L and Zhang LH: Comparison of different endoscopic scoring systems in patients with chronic rhinosinusitis: Reliability, validity, responsiveness and correlation. Rhinology 55: 363-368, 2017.

19. Greguric T, Trkulja V, Baudoin T, Grgic M, Smigovec I and Kalogjera L: Differences in the sino-nasal outcome test 22 and visual analog scale symptom scores in chronic rhinosinusitis with and without nasal polyps. Am J Rhinol Allergy 30: 107-112, 2016.

20. Dietz de Loos DA, Hopkins C and Fokkens WJ: Symptoms in chronic rhinosinusitis with and without nasal polyps. Laryngoscope 123: 57-63, 2013.

21. Livak KJ and Schmittgen TD: Analysis of relative gene expression data using real-time quantitative PCR and the 2(-Delta Delta C(T)) method. Methods 25: 402-408, 2001.

22. Hellquist HB: Nasal polyps update. Histopathology. Allergy Asthma Proc 17: 237-242, 1996.

23. Sedaghat AR: Chronic Rhinosinusitis. Am Fam Physician 96: 500-506, 2017.

24. Campbell K: Contribution of epithelial-mesenchymal transitions to organogenesis and cancer metastasis. Curr Opin Cell Biol 55: 30-35, 2018.

25. Kalluri R and Weinberg RA: The basics of epithelial-mesenchymal transition. J Clin Invest 119: 1420-1428, 2009.

26. Lovisa S, LeBleu VS, Tampe B, Sugimoto H, Vadnagara K, Carstens JL, Wu CC, Hagos Y, Burckhardt BC, PentchevaHoang T, et al: Epithelial-to-mesenchymal transition induces cell cycle arrest and parenchymal damage in renal fibrosis. Nat Med 21: 998-1009, 2015.

27. Sader F, Denis JF, Laref H and Roy S: Epithelial to mesenchymal transition is mediated by both TGF- $\beta$ canonical and non-canonical signaling during axolotl limb regeneration. Sci Rep 9: 1144, 2019.

28. Weiss A and Attisano L: The TGFbeta superfamily signaling pathway. Wiley Interdiscip Rev Dev Biol 2: 47-63, 2013.

29. Darby IA, Zakuan N, Billet F and Desmoulière A: The myofibroblast, a key cell in normal and pathological tissue repair. Cell Mol Life Sci 73: 1145-1157, 2016. 